\title{
The effects of instrumental elliptical polarization on stellar point spread function fine structure
}

Joseph C. Carson, Brian D. Kern, John T. Trauger, James B. Breckinridge

Joseph C. Carson, Brian D. Kern, John T. Trauger, James B. Breckinridge, "The effects of instrumental elliptical polarization on stellar point spread function fine structure," Proc. SPIE 6265, Space Telescopes and Instrumentation I: Optical, Infrared, and Millimeter, 62653M (7 July 2006); doi: 10.1117/12.672518

Event: SPIE Astronomical Telescopes + Instrumentation, 2006, Orlando, Florida , United States 


\title{
The Effects of Instrumental Elliptical Polarization on Stellar Point Spread Function Fine Structure
}

\author{
Joseph C. Carson ${ }^{a, b}$, Brian D. Kern ${ }^{b}$, John T. Trauger ${ }^{b}$, and James B. Breckinridge ${ }^{b}$ \\ ${ }^{a}$ California Institute of Technology, Pasadena, CA, USA; \\ ${ }^{b}$ Jet Propulsion Laboratory, California Institute of Technology, 4800 Oak Grove Dr., Pasadena, \\ CA, 91109, USA
}

\begin{abstract}
We present a status report on a study on the effects of instrumental polarization on the fine structure of the stellar point spread function (PSF). These effects are important to understand because the the aberration caused by instrumental polarization on an otherwise diffraction-limited PSF will likely have have severe consequences for extreme high contrast imaging systems such as NASA's proposed Terrestrial Planet Finder (TPF) mission and the proposed NASA Eclipse mission. The report here, describing our efforts to examine these effects, includes two parts: 1) a numerical analysis of the effect of metallic reflection, with some polarization-specific retardation, on a spherical wavefront; 2) an experimental approach for observing this effect, along with a status report on preliminary laboratory results. The numerical analysis indicates that the inclusion of polarization-specific phase effects (retardation) results in a point spread function (PSF) aberration more severe than the amplitude (reflectivity) effects previously recorded in the literature. Preliminary in-lab results are consistent with our numerical predictions.
\end{abstract}

Keywords: polarization, instrumentation: high angular resolution, methods: analytical, methods: laboratory

\section{INTRODUCTION}

Breckinridge \& Oppenheimer (2004) published a numerical study on the effects of variations in reflectivity from an off-axis $F / 1.5$ parabolic telescope primary mirror on the resulting point spread function (PSF), in the context of high-contrast imaging. That study investigated reflectivity variations across a curved mirror surface due to variations in the angle of incidence for light rays striking different parts of the mirror. Those reflectivity variations are a form of pupil apodization. The authors calculated that these reflectivity variations affected the PSF by a magnitude on the order of $10^{-5}$ of the peak intensity. They noted that this result was particularly important to high contrast imaging experiments such as TPF or Eclipse, which require supression of the on-axis PSF to factors of $\sim 10^{10}$. Hence, a $10^{-5}$ term must be investigated and understood.

Figure 1, from Breckinridge \& Oppenheimer (2004), shows their predicted differences between an ideal PSF from a circular, unapodized, unobscured aperture, and the aperture created by an $F / 1.5$, off-axis, primary mirror. The authors note that the radiation forming the narrow core of the PSF, which establishes the system resolution, reflects from the edge, or the steepest portion of the mirror. This light therefore experiences the greatest polarization attenuation. This phenomenon results in the bifurcated PSF displayed in Figure 1.

This type of bifurcated PSF has been shown experimentally by Fainman \& Shamir (1984) and Kohazi-Kis (2005). In the more recent paper, Kohazi-Kis employs an experimental setup similar to the one described in this paper, where a quickly converging beam (Gaussian-shaped in their case) encounters a flat, reflecting surface. The incident beam is polarized with a polarizing sheet and the outcoming focused PSF is then measured in the original and orthogonal directions. Figure 2, reproduced from that document, shows their measured PSF.

The Figure 2 data results from a setup using two 4-mm diameter focusing lenses (one converges the beam onto the reflecting surface, the other images the resulting PSF) with $50 \mathrm{~mm}$ focal lengths. A $633 \mathrm{~nm} \mathrm{He-Ne}$ laser serves as the light source. In contrast to our experimental setup, described in Section 4, the Kohazi-Kis

Further author information: (Send correspondence to J.C.C.)

J.C.C.: E-mail: Joseph.Carson@jpl.nasa.gov, Telephone: 18183540674

Space Telescopes and Instrumentation I: Optical, Infrared, and Millimeter, edited by John C. Mather, Howard A. MacEwen, Mattheus W.M. de Graauw,

Proc. of SPIE Vol. 6265, 62653M, (2006) -0277-786X/06/\$15 - doi: 10.1117/12.672518

Proc. of SPIE Vol. $626562653 \mathrm{M}-1$ 


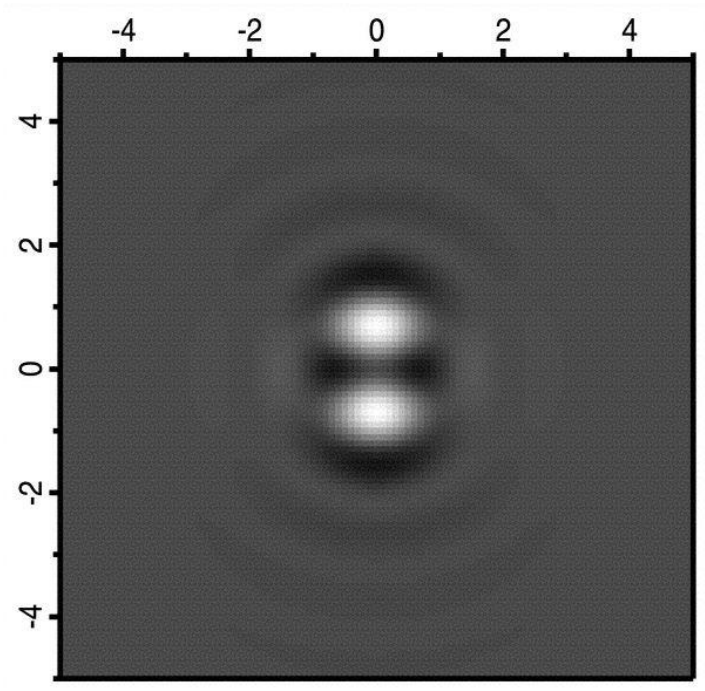

Figure 1. This figure from Breckinridge \& Oppenheimer (2004) shows the difference between an ideal PSF from a circular, unobscured aperture and an $F / 1.5$, off-axis, primary mirror. Axes indicate distance from the image center, in units of $\lambda / \mathrm{D}$.

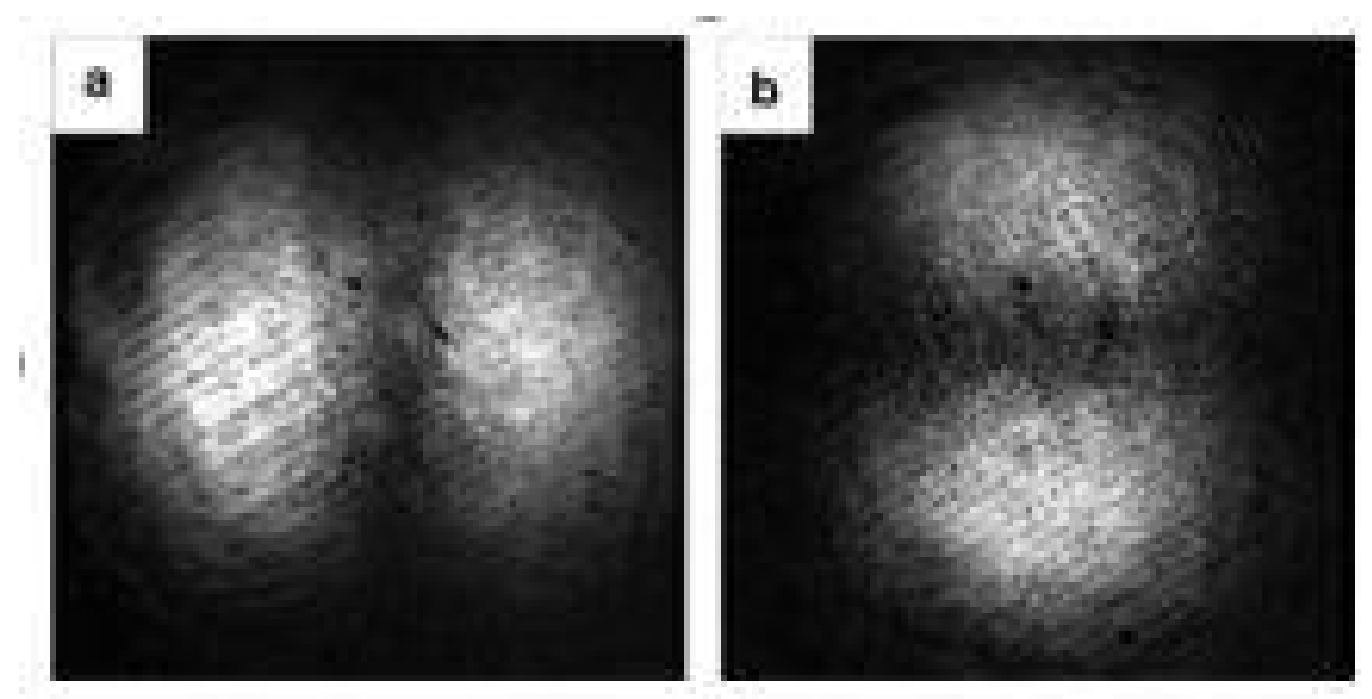

Figure 2. This figure from Kohazi-Kis (2005), reproduced with that author's permission, shows bifurcated PSFs, measured in the lab, resulting from a fast converging beam reflecting off an angled mirror flat. 
setup uses a slower beam $(F / 12.5$ instead of our $F / 2.7)$ and a glass prism as the reflector; our setup employs a protected silver coating currently used for a number of telescope coatings. The Kohazi-Kis result verifies that this bifurcation phenomenon exists. In our experimental study, we hope to also verify this effect, but with measurements for a dielectric surface proposed as a candidate for future NASA space-based telescopes.

Balasubramanian et al. (2005) consider cross-polarization as it may degrade contrast for a TPF coronagraph. In particular, they consider various mirror coatings that may be employed to reduce phase differences that occur between orthogonal polarizations. Their study forms a compliment to ours in that they suggest different coatings that one may test in-lab to try and mitigate these PSF aberrations. We hope that, in the future, we may collaborate with that group to provide laboratory compliments to some of their more analytic considerations.

Our study begins at a similar starting point as the Breckinridge \& Oppenheimer study, regarding the reflection of light off a metallic mirror at a range of angles of incidence. We calculate both the amplitude and phase changes on reflection, for two orthogonal polarizations. In doing so, we conclude that the inclusion of polarization-specific phase effects (retardation) has a greater influence on the resulting PSFs than the amplitude effects (reflectivity) previously calculated. The expected effect should be large enough to be seen in relatively simple laboratory experiments.

Section 2 summarizes the general optical setup we consider. Section 3 describes our numerical predictions. Section 4 describes our experimental setup. Sections 5 presents our conclusions.

\section{GENERAL OVERVIEW OF NUMERICAL AND EXPERIMENTAL TESTS}

We embarked on this study to gain insights into how a large off-axis space-based telescope mirror may, through metallic reflection and polarization-specific retardation, induce aberrations in the imaged stellar point spread function. The numerical and laboratory experiments proposed here do not involve reflection of a plane wave off of a powered metallic surface, but rather reflection of a spherical wave off a tilted flat mirror. The physics of these two configurations is quite similar, in that the angles of incidence of rays across either reflecting surface vary with position, giving rise to variations in reflected amplitude and phase that differ between polarizations. By measuring the resulting PSFs in orthogonal polarizations, the effect should be readily measurable. We are aware of no study that has verified these predictions in the laboratory, with real-life telescope dielectric coatings that may affect these predictions. In particular, we are investigating in-lab the effects of a protected silver coating used by ITT (Rochester, NY) for a number of telescope coatings, which is also a likely candidate for any future TPF or Eclipse mirror coating. The Jet Propulsion Laboratory (JPL) High Contrast Imaging Testbed (HCIT; Trauger et al. 2004) group, of which J. Carson, B. Kern, and J. Trauger are members, is currently approaching in-laboratory high-contrast results where such polarization-induced aberrations may play a role in the final achievable performance. This fact provides a driver for the investigations described in this paper.

Results from the numerical study indicate that inclusion of polarization-specific phase effects (retardation) has a greater influence on the resulting PSFs than the amplitude effects (reflectivity) calculated in Breckinridge \& Oppenheimer (2004). The expected effects should be large enough to be seen in relatively simple laboratory experiments. Preliminary lab results report flux ratios consistent with our numerical predictions; uncertainties are currently too high to verify or contradict the shape of the predicted PSF.

\section{NUMERICAL PREDICTIONS}

The optical train used for the numerical analysis corresponding to this experiment was quite simple: a perfectly spherical converging wavefront reflects off a flat mirror, and propagates to a focus. For simplicity, we define "vertical" as the direction defined perpendicular to the propagation direction of the chief ray, lying in the plane of incidence of the chief ray with the mirror, and assume that the chief ray's angle of incidence is $45^{\circ}$. For each point in a grid in the input pupil, the angle of incidence and plane of incidence is calculated at the flat mirror. The complex reflection coefficients for $p$-polarized (in the plane of incidence) and $s$-polarized (perpendicular to the plane of incidence) light are copied from tabular data supplied by ITT from measurements on the coating in question. The corresponding Jones matrix for each point is tabulated, relating the amplitude and phase of output light in orthogonal linear polarizations to the amplitude and phase of input light in orthogonal linear polarizations. 

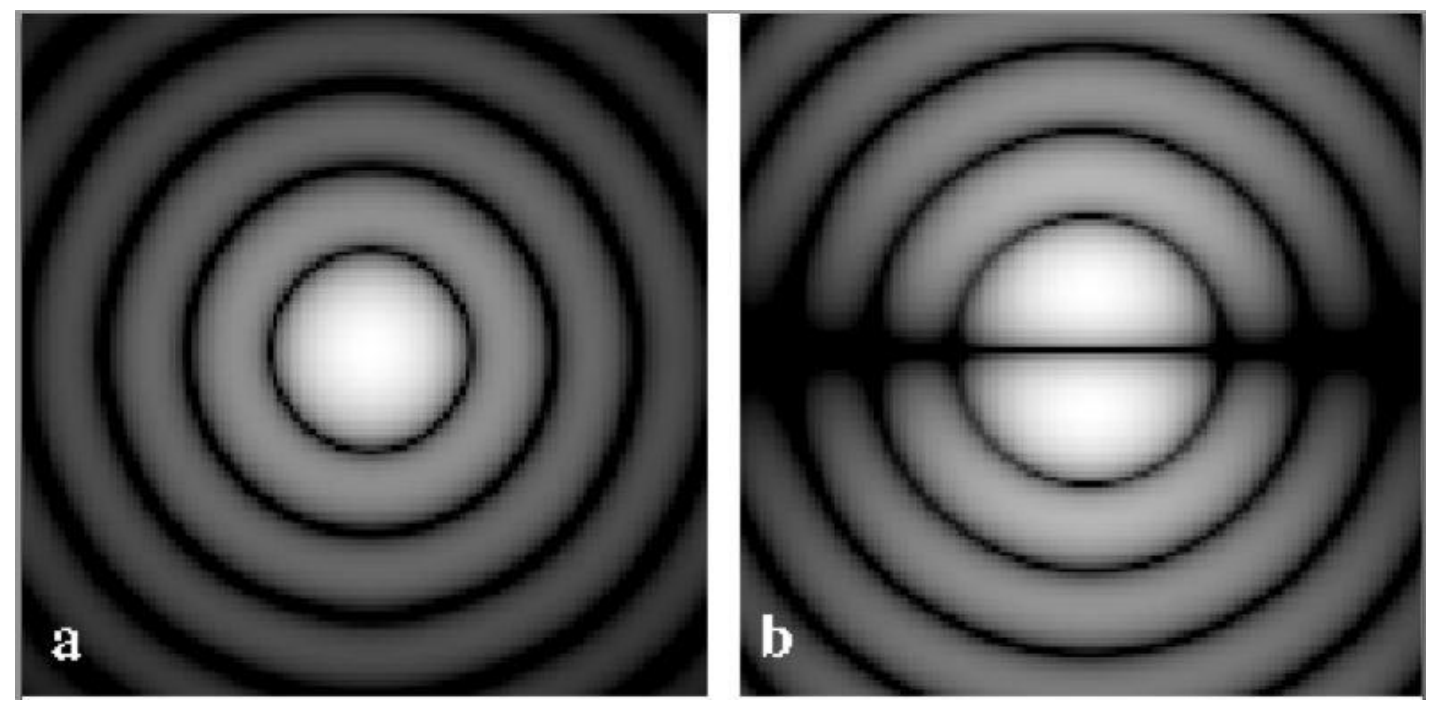

Figure 3. Analytic results for orthogonally polarized PSFs, given a vertically polarized input. Vertical polarization is on the left and horizontal on the right. The images are logarithmically scaled. The intensity in $3 b$ is $\sim 0.003$ that of $1 a$.

If there were no retardation, an input containing only vertically polarized light would produce an output containing only vertically polarized light, and the resulting PSF could be analyzed along the lines of the Breckinridge \& Oppenheimer study. With retardation, the output polarization state depends on the location in the input pupil, and will, in general, be elliptically polarized. By separating the output PSF, which was originally a vertically polarized input, into vertically and horizontally polarized components, the effect of retardation can be directly seen in the horizontally polarized PSF, without having to "subtract off" the unaberrated PSF or make additional differential measurements or theoretical assumptions. The difference between this analysis and traditional ellipsometry is that this analysis integrates the retardation over a number of angles and planes of incidence, and observes in the focal plane of the spherical wavefront.

For an input $F / 3$ beam at $635 \mathrm{~nm}$, using the protected silver coating from ITT, we predict that the "crosspolarized" PSF (the outputted horizontal polarization, given a 100\% vertical input) has 1/360 the intensity of the orthogonally polarized PSF. The predicted PSFs in orthogonal polarizations, for a vertically polarized input, are shown in figures $3 \mathrm{a}$ and $3 \mathrm{~b}$.

\section{THE LABORATORY EXPERIMENT}

Our laboratory setup includes two inter-changeable configurations, as shown in Figure 4. In both configurations, a standard, commercially available polarizing sheet (with polarization leakage $\sim 0.1 \%$ ) linearly polarizes the light emerging from a 5-micron pinhole. We orient the polarizing sheet parallel to the vertical, to maximize detection of the cross-polarization effect. Next, an iris constrains the beam f-number while also minimizing stray light. A following aspheric lens (effective diameter $=4 \mathrm{~mm}$, focal length $=11 \mathrm{~mm}$ ) converges the light into a fast beam, $F / 2.7$ in our case. Next the light either reflects off a silver coated (ITT [Rochester, NY]) flat set at a 45-degree angle, or continues on a straight-line setup. In either case, a downstream aspheric lens (also $4 \mathrm{~mm}$ diameter and $11 \mathrm{~mm}$ focal length) focuses the emerging light onto a CCD camera (from Apogee). Before the beam reaches the CCD camera, a calcite displacement polarizer (from Karl Lambrecht Corporation) splits the beam so that PSFs with orthogonal polarizations image simultaneously on the detector chip.

The straight-line setup serves as a calibration comparison: PSF structure seen in the reflective setup may be compared with the straight-line setup to verify that the mirror flat is indeed the source of an observed fine-structure effect. The particular selection of the lenses fulfills several requirements: the existence of a fast beam incident on the mirror ensures that the cross-polarization effect is maximized. The pinhole-image demagnification, caused by the first lens, ensures that the pinhole is unresolved compared to the imaged beam 


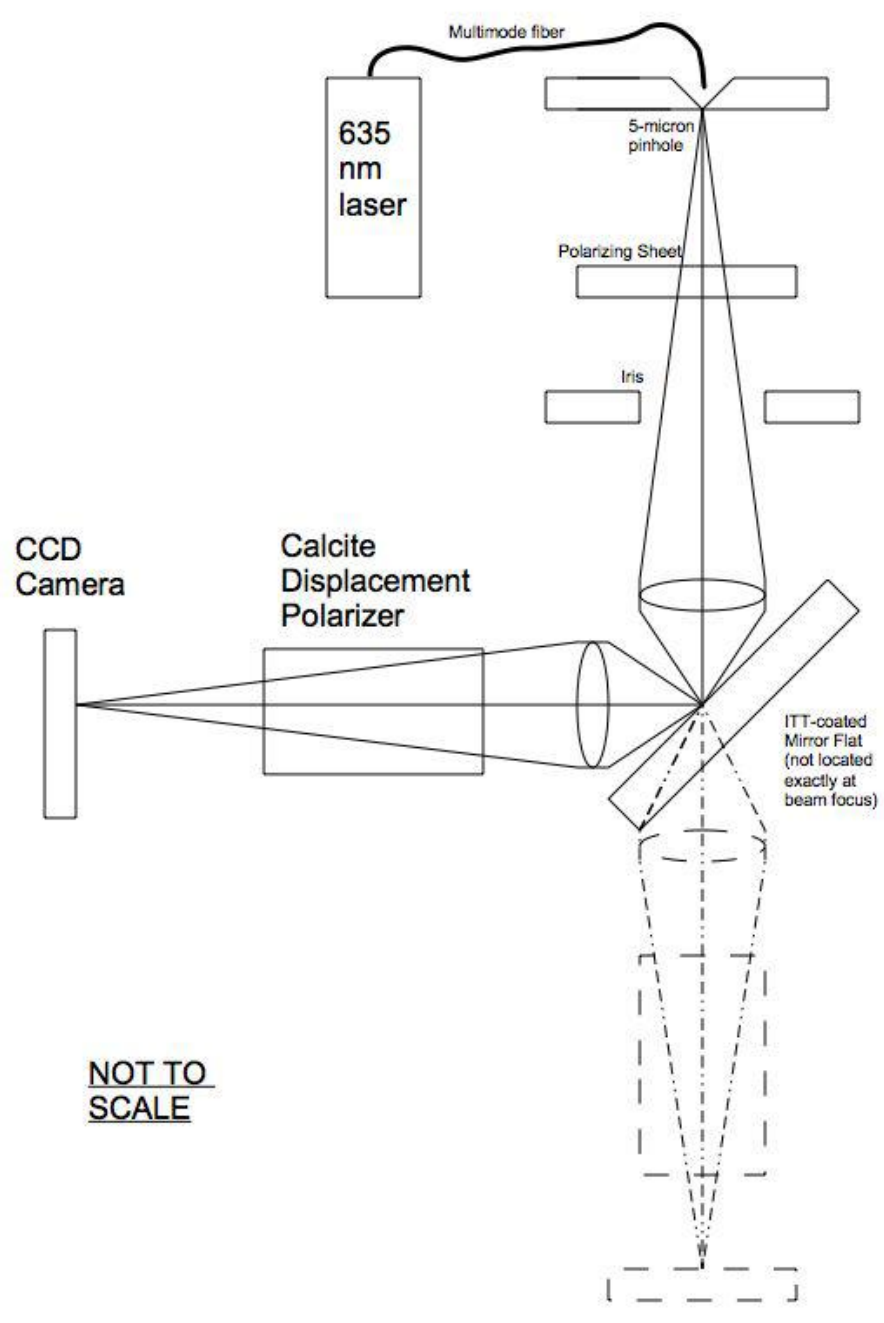

Figure 4. This schematic outlines the laboratory setup. Solid rays indicate the reflective configuration. Dashed beam rays indicate the straight-through setup we use as a comparison calibration. The fast converging beams represent $F / 2.7$. Both lenses (aspherics) have $4 \mathrm{~mm}$ effective diameters and $11 \mathrm{~mm}$ focal lengths. The lenses were selected and positioned to create a fast-beam (making the cross-polarization effect easy to recognize), allow for a well-sampled PSF at the CCD, and ensure an unresolved pinhole (thus mimicing a stellar point source). 

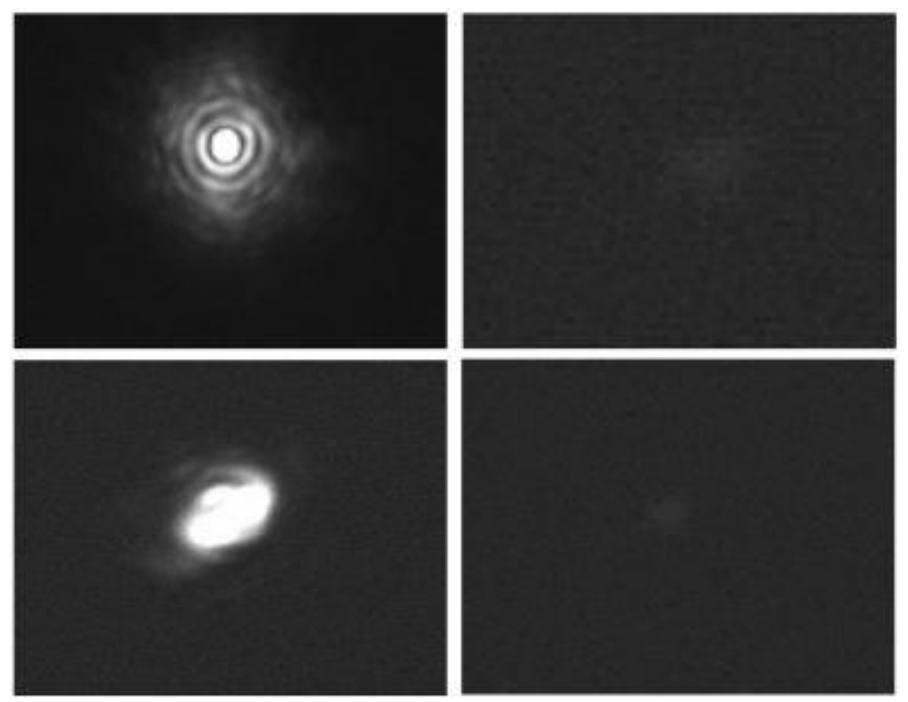

Figure 5. Experimental results for orthogonally polarized PSFs, given a vertically polarized input. Vertical polarization is on the left and horizontal is on the right. The top images represents "straight-line" data and the bottom images represent "reflective" data, as defined in Figure 4. The very faint PSF that may just be discerned in the upper right image represents leakage from our polarizing sheet. The bottom-right PSF, also marginally visible, represents a combination of this leakage (about 1 part in 1000) along with the cross-polarization term induced by the convergent beam reflecting off the mirror flat. The images are plotted on a linear scale. The cross-polarized PSF for the "reflective" setup measures $0.4 \% \pm 0.3 \%$ of the vertical polarization PSF (after polarizing sheet leakage has been removed).

diffraction pattern. Thus it effectively mimics a point source. The position and focal length of the second lens ensures that the transmitted beam successfully focuses on the CCD camera, and with an appropriately sampled pixel scale. The top half of Figure 5 shows the orthogonal PSFs for the straight-line setup. The reflective setup PSF, noticeably messier (as can be seen in the two bottom images in Figure 5) contains too many un-accounted for aberrations (perhaps a mixture of astigmatism, coma, and stray light) to produce a meaningful test of the PSFs predicted bifurcated shape. The flux level of the cross-polarized PSF, $0.4 \% \pm 0.3 \%$ of the orthogonal PSF intensity (after polarizing sheet leakage has been removed) is consistent with our numerical predictions.

\section{CONCLUSIONS}

We have presented procedures and results from a numerical and laboratory study testing for the effects of instrumental elliptical polarization on stellar point spread function fine structure. Our numerical experiments indicate that polarization-specific phase effects (retardation) have a larger effect on PSF aberrations than the amplitude (reflectivity) effects previously calculated by Breckinridge \& Oppenheimer (2004). Our preliminary experimental results are consistent with this prediction.

\section{ACKNOWLEDGMENTS}

The research described in this paper was carried out at the Jet Propulsion Laboratory, California Institute of Technology, under a contract with the National Aeronautics and Space Administration.

\section{REFERENCES}

1. Balasubramanian, K., Hoppe, D. J., Mouroulis, P. Z., Marchen, L. F., \& Shaklan, S. B. 2005, SPIE 5905, $59050 \mathrm{H}$

2. Breckinridge, J. B. \& Oppenheimer, B. R. 2004, ApJ 600, 1091

3. Kohazi-Kis, A. 2005, Optics Communications 253, 28 
4. Trauger, J., Burrows, C., Gordon, B., Green, J., Lowman, A., Moody, D., Niessner, A., Shi, F., \& Wilson, D. 2004, SPIE 5487, 1330 